\title{
Microstructural Simulations of the Influence of Solidification Velocity on Freckle Initiation during Directional Solidification
}

\author{
Lang YUAN and Peter D. LEE \\ Department of Materials, Imperial College London, SW7 2AZ, London, UK. \\ E-mail: lyuan@imperial.ac.uk; p.d.lee@imperial.ac.uk
}

(Received on May 26, 2010; accepted on August 5, 2010)

\begin{abstract}
The initiation of freckles by thermosolutal convection during the directional solidification of $\mathrm{Pb}-\mathrm{Sn}$ alloys was studied numerically at the microstructural level. The model predicts the detailed dendritic structures, microsegregation and interdendritic convection in three dimensions. The onset and sustained growth of solutal channel (freckles) was simulated as a function of casting speed. The predictions were compared with experimental measurements via the introduction of a Rayleigh number. Good agreement was achieved, suggesting that such models might be used to predict the critical Rayleigh number for more complex alloy systems with further development. It was found that the large density variation of $\mathrm{Pb}-\mathrm{Sn}$ alloys induces strong upward convection of segregated liquid and promotes the formation of solutal channels. However, the competition between upward solute transport and dendritic growth determines both the initiation and sustained growth of these channels. These open solute channels become the defect structure known as a freckle upon final solidification.
\end{abstract}

KEY WORDS: numerical simulation; directional solidification; interdendritic convection; freckles; dendritic microstructures; solutal convection.

\section{Introduction}

Freckles are severe channel-like segregates commonly observed in directionally solidified or single-crystal castings of Ni-base superalloys. ${ }^{1,2)}$ The existence of freckles has a deleterious impact on mechanical properties of critical components such as turbine blades and discs in gas-turbine engines. ${ }^{3)}$ There have been a number of prior experimental studies suggesting that freckles are initiated by thermosolutal convection in the mushy zone. ${ }^{4-9)}$ A variety of mathematical models have been proposed to predict the formation of freckles. All of these models can be classified into two groups: one based on the use of mathematical criteria ${ }^{10-14)}$ and the other based on solution of mass, momentum, energy, and species conservation equations. ${ }^{15-20)}$ However, both classes of models simulate the flow at a macroscopic level, rather than studying the initial stages of channel formation at a microstructural level.

The most common mathematical criteria used is the Rayleigh number, a dimensionless value which describes convective fluid instability. Different forms of the Rayleigh number have been proposed to characterize freckle formation, as reviewed by Yang et al. ${ }^{14)}$ Beckermann et al. ${ }^{11,12)}$ examined the possible definitions and found a definition proposed by Worster ${ }^{21)}$ to be physically most meaningful, which accounts for the resistant drag force from structure (permeability) and the driven force from density variation. It has the form:

$$
\mathrm{Ra}_{h}=\frac{g \bar{K} h}{\alpha \nu}\left(\frac{\Delta \rho}{\rho_{0}}\right)
$$

where $h$ is the characteristic length scale, $g$ is the acceleration due to gravity, $\bar{K}$ is the mean permeability of the mushy zone, $\alpha$ is thermal diffusivity, $v$ is kinematic viscosity, and $\Delta \rho / \rho_{0}$ is the relative density inversion due to thermal and/or compositional variation. The calculated Rayleigh numbers were compared to previous experimental data and the results have shown a good evaluation of freckle formation for $\mathrm{Pb}-\mathrm{Sn}$ alloys. The Rayleigh number provides an efficient means of evaluating stability for freckle formation by considering the average solidification environment. However, the form of the Rayleigh number varies with different alloys systems and the determination of the critical value depends on a wide range of experimental studies and hence is always empirically determined.

A number of authors have presented macroscopic numerical models which can directly predict the formation of segregated solutal channels due to thermosolutal buoyancy force. Felicelli et al. ${ }^{22)}$ developed a mathematical model of macrosegregation in $2 \mathrm{D}$ to study the segregated channel formation of $\mathrm{Pb}-10 \mathrm{wt} \% \mathrm{Sn}$ alloy. The prediction of channels in directional solidification is the first of its kind and shows the correct qualitative behaviour observed in experiments. ${ }^{6,23)}$ Such models have now been extended to study multicomponent alloy systems and also in $3 \mathrm{D} .{ }^{16,17,22,24)}$ However, the initiation of the solutal instabilities in such models depends on either inherent noise in the numerical 
method or applied noise. Since segregation starts at microscale, the rejection of solute from the growing dendrites is the key driving force, while their morphologies limit interdendritic thermosolutal convection. Therefore, a microscale solidification model that provides this information can be expected to give a better understanding of the instabilities of the interdendritic convection and also the associated channel formation.

In this paper, a microscale numerical model, which simulates the morphologies of dendrites, microsegregation and interdendritic convection due to variations of thermal and solutal distributions, was developed to study the onset of freckling of $\mathrm{Pb}-\mathrm{Sn}$ alloy in the interdendritic region during directional solidification in 3D. The model theory is summarized first, followed by the calculation of the Rayleigh number which was used to evaluate the solidification conditions. Four cases with different casting speeds were simulated to investigate the solute channel formation and also the influence of casting speed on the tendency for freckling.

\section{Model Theory}

An existing open source numerical model of dendritic solidification without fluid flow ${ }^{25-29)}$ was first extended to simulate forced convection ${ }^{30-32)}$ and in this study to incorporate thermal and solutal buoyancy flows. The details of the model implementation are given in these prior publications and in the freely available code and documentation (www.imperial.ac.uk/advancedalloys). Therefore, only the key equations are described below. It is assumed that the liquid is incompressible and the growth of the solid/liquid interface is determined by solute diffusion and solute partition subjected to the equilibrium conditions. The governing equations for mass, momentum, energy and solute are given by:

$$
\begin{aligned}
& \nabla \cdot\left(f_{1} \vec{u}\right)=0 \\
& \frac{\partial}{\partial t}\left(f_{1} \vec{u}\right)+\nabla \cdot\left(f_{1} \vec{u} \vec{u}\right)-\nabla \cdot\left[\frac{\mu}{\rho} \nabla\left(f_{1} \vec{u}\right)\right]=-\frac{f_{1}}{\rho} \nabla P+S \\
& \frac{\partial T}{\partial t}+\nabla \cdot(\vec{u} T)=\frac{\lambda}{\rho c_{\mathrm{p}}} \nabla^{2} T-\frac{L}{c_{\mathrm{p}}} \frac{\partial f_{1}}{\partial t} \\
& \frac{\partial C_{\mathrm{e}}}{\partial t}+\nabla \cdot\left(\vec{u} C_{1}\right)=\nabla \cdot\left(D_{\mathrm{e}} \nabla C_{1}\right)
\end{aligned}
$$

where $\vec{u}$ is the velocity vector in liquid, $P$ is the pressure, $\mu$ is the viscosity, $c_{\mathrm{p}}$ is specific heat, $\lambda$ is thermal conductivity, $f_{1}$ is the liquid fraction, $L$ is the latent heat, $C_{\mathrm{s}}$ and $C_{1}$ are the average solute concentrations of the solid and liquid. $S$ is the source term, accounting for buoyancy forces. A Boussinesq approximation was used for both the temperature and composition dependency of density:

$$
S=g\left(1-\beta_{\mathrm{T}}\left(T-T_{\mathrm{ref}}\right)-\beta_{\mathrm{c}}\left(C-C_{\mathrm{ref}}\right) .\right.
$$

where $\beta_{\mathrm{T}}$ and $\beta_{\mathrm{c}}$ are the thermal and solutal expansion coefficients, respectively.

$C_{\mathrm{e}}$ and $D_{\mathrm{e}}$ are equivalent concentration and solute diffusion coefficient, respectively. They are defined as:
Table 1. Thermophysical properties of $\mathrm{Pb}-10 \mathrm{wt} \% \mathrm{Sn}$.

\begin{tabular}{llll}
\hline Property & Variable & Value & Unit \\
\hline Partitioning coefficient & $k$ & 0.31 & - \\
Liquidus slope & $m$ & -2.33 & $\mathrm{~K} / \mathrm{wt} \%$ \\
Density & $\rho$ & $10.13 \times 10^{3}$ & $\mathrm{~kg} / \mathrm{m}^{3}$ \\
Solutal expansion coefficient & $\beta_{c}$ & $5.15 \times 10^{-3}$ & $\mathrm{wt} \%^{-1}$ \\
Thermal expansion coefficient & $\beta_{\mathrm{T}}$ & $1.21 \times 10^{-4}$ & $\mathrm{~K}-1$ \\
Melting point of Pb & $T_{\mathrm{m}}$ & 600 & $\mathrm{~K}$ \\
Latent Heat & $L$ & $3.76 \times 10^{5}$ & $\mathrm{~J} / \mathrm{kg}$ \\
Specific heat & $c_{\mathrm{p}}$ & 167 & $\mathrm{~J} / \mathrm{kg} \cdot \mathrm{K}$ \\
Eutectic temperature & $T_{\text {eut }}$ & 456 & $\mathrm{~K}$ \\
Eutectic concentration & $C_{\text {eut }}$ & 61.9 & $\mathrm{wt} \% \mathrm{Sn}$ \\
Dynastic viscosity & $\mu$ & $2.47 \times 10^{-7}$ & $\mathrm{~m}{ }^{2} / \mathrm{s}$ \\
Thermal diffusivity & $\alpha$ & $1.1 \times 10^{-5}$ & $\mathrm{~m}{ }^{2} / \mathrm{s}$ \\
Solutal diffusivity in liquid & $D_{\mathrm{L}}$ & $3 \times 10^{-9}$ & $\mathrm{~m}{ }^{2} / \mathrm{s}$ \\
Solutal diffusivity in solid & $D_{\mathrm{S}}$ & $2 \times 10^{-13}$ & $\mathrm{~m}^{2} / \mathrm{s}$ \\
\hline & & & \\
\hline
\end{tabular}

$$
\begin{gathered}
C_{\mathrm{e}}=C_{1} f_{1}+C_{\mathrm{s}} f_{\mathrm{s}} \cdots \\
D_{\mathrm{e}}=D_{1} f_{1}+k \cdot D_{\mathrm{s}} f_{\mathrm{s}}
\end{gathered}
$$

With the definitions above, Eq. (5) can be applicable to represent the solute diffusion in the entire domain, including the solid, liquid and interfacial regions. ${ }^{32)}$ Once the solute concentration is updated by solving Eqs. (2) to (5), the change of fraction solid can be derived from Eq. (7), giving as:

$$
\frac{\partial f_{\mathrm{s}}}{\partial t}=\frac{1}{C_{1}(1-k)}\left[-\frac{\partial C_{\mathrm{e}}}{\partial t}\left[1-(1-k) f_{\mathrm{s}}\right] \frac{\partial C_{1}}{\partial t}\right] \ldots
$$

This equation is used to correlate the growth rate in a growing cell. When $\Delta f_{\mathrm{s}}$ is less than 0 , it means the cell is remelting. The anisotropy in interfacial energy of the solid-liquid interface is imposed using a modified decentered square/octahedron algorithm. ${ }^{29,33)}$ This algorithm propagates the interface in terms of the change of solid fraction (calculated by Eq. (9)) and ensures the dendrites grow with preferred $\langle 100\rangle$ direction.

The convection and diffusion equations are solved on the same regular spatial grid. A projection algorithm ${ }^{34)}$ based on the staggered mesh is applied to solve the Navier-Stokes equations by using the finite volume method. A preconditioned conjugate gradient method (PCG) is used to solve the pressure Poisson equation. The flow velocity in the mushy zone is dampened proportionally to the fraction solid. A linearized $\mathrm{Pb}-\mathrm{Sn}$ phase diagram was used in this study with constant partition coefficient and liquidus slope. Thermophysical properties of $\mathrm{Pb}-10 \mathrm{wt} \% \mathrm{Sn}$ alloy are listed in Table 1.

\section{Rayleigh Number}

Although the model directly predicts solute channel onset, calculating the Rayleigh number is useful for comparison of the predictions to prior experimental studies. The version recommended by Ramirez et al. ${ }^{12)}$ was used here (Eq. (1)). The methods used for the calculation of length 


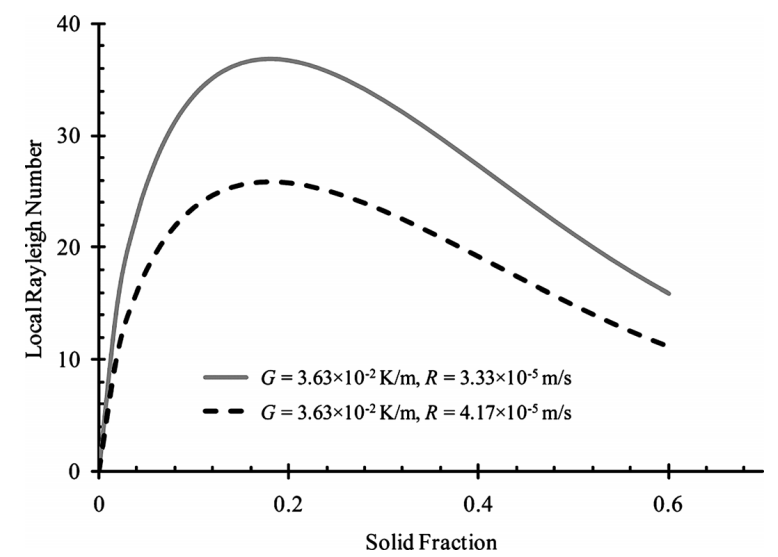

Fig. 1. Local variation of Rayleigh numbers for $\mathrm{Pb}-\mathrm{Sn}$ with two solidification conditions.

scale, permeability and density inversion are identical to their work. Although the microstructural model predicts the primary spacing, it is varies dramatically depending on the solutal flow. Therefore an empirical relationship for primary arm spacing was used for consistent comparison with experiment ${ }^{14)}$ :

$$
\lambda_{1}=921.7 C_{0}^{0.134} G^{-0.354} R^{-0.261}
$$

where $C_{0}$ is the initial concentration in weight percent, $G$ is the temperature gradient in $\mathrm{K} / \mathrm{cm}$ and $R$ is the casting speed in $\mu \mathrm{m} / \mathrm{s}$.

Since the density inversion and permeability vary throughout the mush, a local Rayleigh number was calculated as shown in Fig. 1 for a $\mathrm{Pb}-10 \mathrm{wt} \% \mathrm{Sn}$ alloy for two cases: $1 . \quad G=3.63 \times 10^{-2} \mathrm{~K} / \mathrm{m}, R=3.33 \times 10^{-5} \mathrm{~m} / \mathrm{s}$ and 2 . $G=3.63 \times 10^{-2} \mathrm{~K} / \mathrm{m}, R=4.17 \times 10^{-5} \mathrm{~m} / \mathrm{s}$. A maximum value across the mushy zone can be identified in both curves and occurs at an average solid fraction between 0.15 and 0.2 ; hence this is the most likely point where solutal driven flow will overcome the viscous dampening forces. Therefore, this single value was used as the representative Rayleigh number to evaluate each solidification condition.

\section{Results and Discussion}

\subsection{Simulation Conditions}

A domain $7.5 \mathrm{~mm}$ high, $\sim 5$ primary dendrites wide and one deep was used to investigate the solute channel formation at a microstructural level (Fig. 2). To initiate the microstructure, 3-5 seeds were fixed in a straight row on the base of the domain spaced at the steady state primary dendritic arm spacing given by Eq. (10). A relatively large cell size of $15 \mu \mathrm{m}$ was used to reduce the total number of cells in the domain to $\sim 1$ million yet remain fine enough to resolve the segregation from the secondary dendritic arms.

A constant temperature gradient $(G)$ was applied to the domain with a constant casting speed $(R)$. Periodic boundary conditions were used for velocities and solute concentrations on all four side surfaces, while at the top and bottom surfaces a zero-flux boundary condition was applied. Four cases were simulated to investigate solute channel initiation as a function of casting velocity ranging from $3.3 \times$ $10^{-6}$ to $3.3 \times 10^{-4} \mathrm{~m} / \mathrm{s}$. The solidification conditions are given in Table 2. The maximum Rayleigh number for each

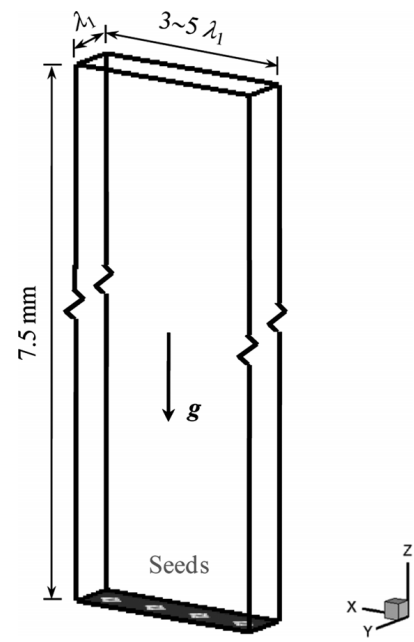

Fig. 2. Schematic of the directional solidification system with $3-5$ seeds preset at the bottom.

Table 2. Solidification conditions of $\mathrm{Pb}-10 \mathrm{wt} \% \mathrm{Sn}$ alloys for numerical simulations.

\begin{tabular}{lllll}
\hline Case & $C_{0}, \mathrm{wt} \% \mathrm{Sn}$ & $G \times 10^{-2}, \mathrm{~K} / \mathrm{m}$ & $R \times 10^{6}, \mathrm{~m} / \mathrm{s}$ & $\mathrm{Ra}$ \\
\hline 1 & 10 & 3.63 & 330 & 1.2 \\
2 & 10 & 3.63 & 41.7 & 25.7 \\
3 & 10 & 3.63 & 33 & 36.1 \\
4 & 10 & 3.63 & 3.3 & 1193.6 \\
\hline
\end{tabular}

case was also calculated, spanning 3 orders of magnitude.

For all cases, the calculation of thermosolutal convection is turned on after the dendrites have grown approximately $2 \mathrm{~mm}$ in length. This was done to avoid the artificial seeding from influencing flow.

\subsection{Solute Channel Formation}

The simulated morphologies of dendrites with different casting speeds, together with $\mathrm{Sn}$ concentration profiles and velocity vectors on $x-z$ plane, are shown in Fig. 3. Vertical channels can be found in all cases. During solidification, the lighter $\mathrm{Sn}$ was rejected into the interdendritic region and advected into the bulk. This upward convection transports the lighter interdendritic liquid into the fully molten region in the form of plumes, which produce a swirling flow ahead of the dendrites which can be observed in all cases (Fig. 3). Because the solute diffusivity of the liquid is much lower than the thermal diffusivity (the Lewis number is approximately 3600 ), the lighter liquid retains its composition as it flows upward through the mush into regions of higher temperature. This redistributes the solute in the solidifying region and may lead to macrosegregation. Since the segregated solute has higher Sn concentration, it can decrease the local undercooling and, thereby, retard the growth of dendrites. As a consequence, the secondary arms in the channel can remelt, potentially sustaining the formation of the vertical channels. In cases 1-3, the channels were not self-sustaining, with their formation being terminated by fast growing secondary arms. Tertiary arms then grow out of these secondaries, trying to re-establishing a primary spacing closer to the initial value. From the morphology of the dendrites, it can be seen that there is a strong potential for channels, but there is an equally strong potential for an- 


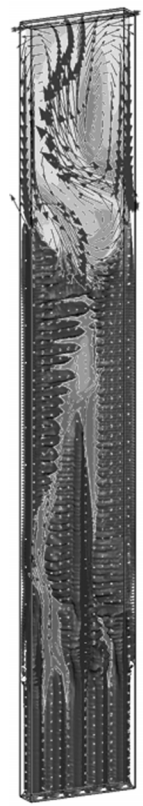

(a)

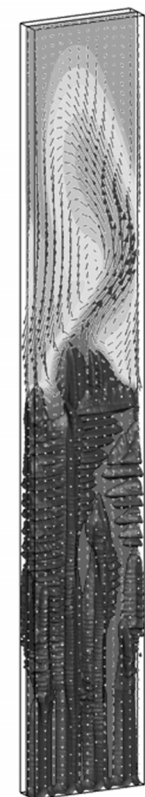

(b)

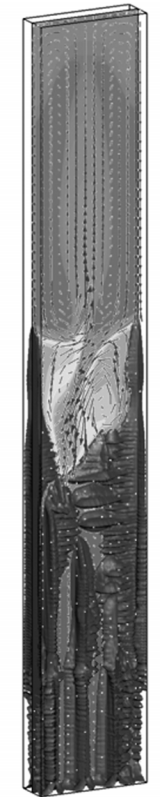

(c)

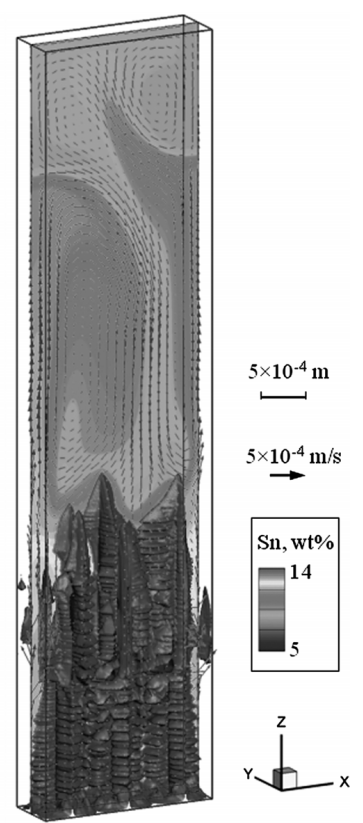

(d)
Fig. 3. Simulated dendritic morphologies, Sn concentration profiles and velocity vectors on $x-z$ plane with different casting speeds: (a) case $1, t=78 \mathrm{~s}$, (b) case $2, t=170 \mathrm{~s}$; (c) case $3, t=140 \mathrm{~s}$; and (d) case $4 t=300 \mathrm{~s}$.

other dendrite to grow into it, i.e. self-sustaining solute channels (the initiation of freckles) are not formed.

From Fig. 3(a), the high solute concentration in the channel significantly prohibited the growth of dendrite arms in the region and also fed the upward convection in the plume. However, much stronger convection occurred above the dendrites, and caused mixing of the solute which was transported from interdendritic region. This convection prohibited the development of the dendrite in the middle of the domain and also promoted the growth the lateral dendrite arms. The growth of the secondary arms from one lateral dendrite stopped the development of the plume. It can be shown that the solute velocity in the channel was less than $3 \times 10^{-4} \mathrm{~m} / \mathrm{s}$ which is smaller than the casting speed for this case $\left(3.3 \times 10^{-4} \mathrm{~m} / \mathrm{s}\right)$. One possible reason for the formation of a freckle channel is that the intrinsic velocity of the interdendritic liquid is faster than the dendritic growth velocity. For case 1, even without the help of bulk convection, the solute channel does not survive, consistent with prior experiments for similar Rayleigh numbers/conditions. ${ }^{6}$ ) Similar phenomena occurred in cases 2 and 3 (Figs. 3(b) and 3(c)). Very early on during the solidification, a high Sn concentration is reached in the interdendritic channels. However, there the upward flow exhibited in these channels is very weak $\left(\sim 10^{-5} \mathrm{~m} / \mathrm{s}\right)$ and a sustained recirculation within the dendrites is not formed.

In case 4 (Fig. 3(d)), a self sustaining channel forms with a strong upward flow and recirculating downwards flows all within the dendritic structure, as well as within the bulk. The initiation location of the channels is effectively random and here it occurred at the periodic boundary, the results are re-plotted in Fig. 4 with a half domain shift applied in the $x$ direction. Streamlines were also added to the plot to show the recirculation occurring within the mush. A vertical channel, of about one dendrites width, remains open to the

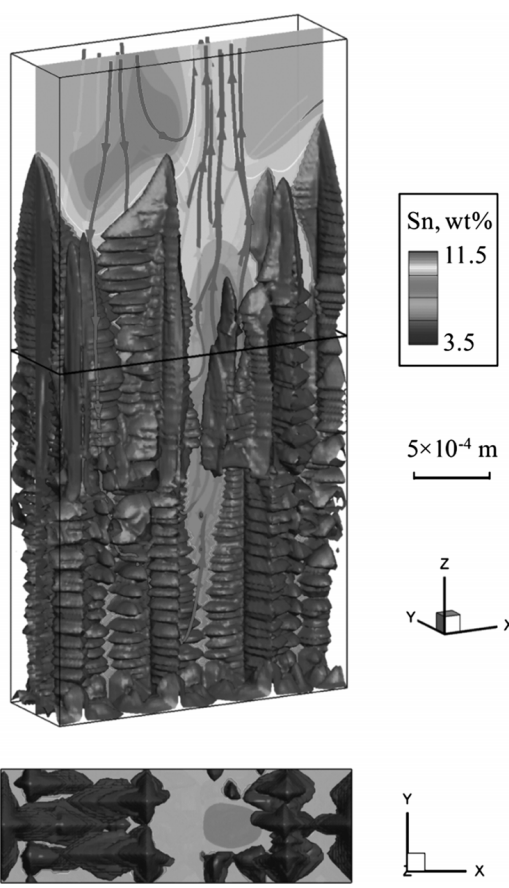

Fig. 4. Flow patterns in and around the vertical solutal channel shown in Fig. 3(d) (Re-plotted by applying a half domain shift in the $x$ direction).

bulk (shown by the upward streamlines). This is feed by downward flows in a number of neighbouring interdendritic spaces. The gradually built solute layer in the channel ensures the continuous upward flow and higher level of solute concentration was maintained at the mouth of the channel, keeping the channel open. Therefore, a self-sustained channel was formed in this case, which is able to develop into a freckle after solidifying. In addition, the measurements of the upward velocity in the channel were in the order of $10^{-5} \mathrm{~m} / \mathrm{s}$, almost three times greater than the casting speed of $3.3 \times 10^{-6} \mathrm{~m} / \mathrm{s}$, which also assists the formation of solute channels. $^{20)}$

The assessment of prior $\mathrm{Pb}-\mathrm{Sn}$ experiments has suggested a critical value of the Rayleigh number is in the range of 38.8 to 40.3 (38 to 46 by Ramirez et al. ${ }^{12)}$ ). In the current study, as discussed above, no open channels are formed in cases 1-3 which all have a Rayleigh number below 38, although case 3 is close with a maximum Ra of 36. However, in case 4 where a self-sustaining channel forms, the maximum Rayleigh number is $\sim 1000$, much larger than the experimental critical value of $\sim 40$. This suggests that the present model might be used to predict the critical Rayleigh number for freckle formation for a range of solidification conditions and also potentially for different alloy systems.

\subsection{Effects of Casting Speed}

The Rayleigh number has been validated as a good indicator for freckle formation. Thereby, it is plotted versus cast speed in Fig. 5. The initial Sn concentration and temperature gradient remain constant $\left(10 \mathrm{wt} \%\right.$ and $3.63 \times 10^{2} \mathrm{~K} / \mathrm{m}$, respectively). As expected, the faster the casting speed, the lower the Rayleigh number. This implies that freckles tend to form at low casting speed. In terms of the critical Rayleigh number, the highest casting speed for freckling is 


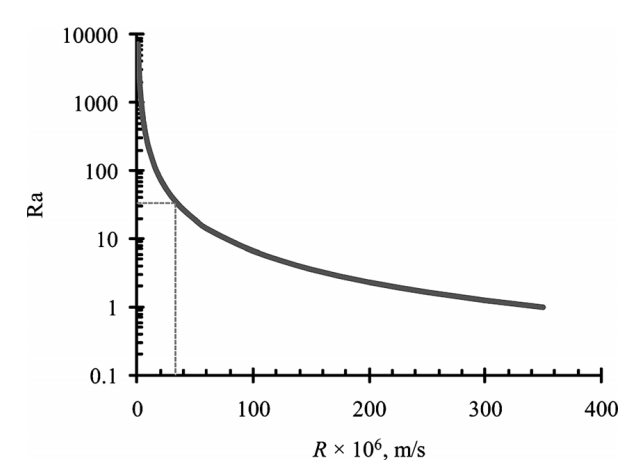

Fig. 5. Variation of the Rayleigh number with casting speed for $G=3.63 \times 10^{2} \mathrm{~K} / \mathrm{m}$ and $C_{0}=10 \mathrm{wt} \%$.

$\sim 3.5 \times 10^{-5} \mathrm{~m} / \mathrm{s}$ under the specified solidification conditions.

In terms of simulations, the lower growth speed can decrease the rate of solute rejection, which results in a decrease in the concentration gradient in the interdendritic region. It can be seen that the convection in case 4 was weaker in the melt bulk when compared with case 1. However, the slow casting speed results slow growth of the dendrites. The competition between the upward solute transport and dendritic growth determine the existence of the solute channels. In addition, the low growth speed of dendrites allows the segregated liquid more time to develop the flow and to redistribute the solute. In both cases 2 and 3, the interdendritic flow velocity is the same order as the casting speed. They can be regarded as borderline situations for freckle formation (note their Ra numbers are 26 and 36, getting close to the critical value). This suggests that reducing the casting speed further (i.e. case 4) could result in freckling.

\section{Conclusions}

The solute channel formation of $\mathrm{Pb}-\mathrm{Sn}$ alloys in directional solidification conditions was studied by a microscale solidification model with detailed examination of dendritic morphologies, microsegregation and interdendritic convection. The simulated results have shown good agreement with experimental measurements in terms of the maximum Rayleigh number. It has been found that:

(1) Although the large density variation in $\mathrm{Pb}-\mathrm{Sn}$ alloys encourages thermosolutal convection, the competition between upward solute transport and dendritic growth determines the initiation and sustained growth of solute channels, and hence freckle formation.

(2) The convective flow of solute enriched interdendritic liquid can cause remelting of dendrites resulting in the formation of an open solute channel, forming a freckle defect upon solidification.

(3) A high casting speed prohibits the formation of freckles. It causes strong thermosolutal convection at the solidification front but also fast growth of the dendrites. The low permeability in the interdendritic region (due to fast dendritic growth) prevents the solute from sustaining a continuous upward plume that results in a freckle during solidification. For a given gradient, as the casting speed decreases the dendritic spacing increases, increasing the per- meability (and Rayleigh number) making self-sustaining thermal-solutal plumes within the mushy zone more favourable. The model therefore predicts the experimentally observed correlation between Rayleigh number and freckle formation.

\section{Acknowledgements}

The authors would like to acknowledge the EPSRC (EP/D50502 and EP/D04619X), Corus, Special Metals and Rolls-Royce for project support.

\section{REFERENCES}

1) A. F. Giamei and B. H. Kear: Metall. Trans., 1 (1970), 2185.

2) T. M. Pollock and W. H. Murphy: Metall. Mater. Trans. A, 27A (1996), 1081.

3) P. D. Genereux and C. A. Borg.: Proc. Int. Symp. Superalloys, ed. by T. M. Pollock, R. D. Kissinger, R. R. Bowman, TMS, Warrendale, PA, (2000), 19.

4) M. Bergman, D. Fearn and J. Bloxham: Metall. Mater. Trans. A, 30 (1999), 1809.

5) R. Grugel and L. Brush: JOM, 49 (1997), 26.

6) J. R. Sarazin and A. Hellawell: Metall.Trans. A, 19A (1988), 1861.

7) N. Streat and F. Weinberg: Metall. Mater. Trans. B, 7 (1976), 417.

8) S. N. Tewari and R. Shah: Metall. Mater. Trans. A, 27A (1996), 1353.

9) L. Wang, V. Laxmanan and J. Wallace: Metall. Mater. Trans. A, 19 (1988), 2687.

10) P. Auburtin, T. Wang, S. L. Cockcroft and A. Mitchell: Metall. Mater. Trans. B, 31 (2000), 801.

11) C. Beckermann and W. J. Boettinger: Metall. Mater. Trans. A, 31A (2000), 2545.

12) J. C. Ramirez and C. Beckermann: Metall. Mater. Trans. A, 34A (2003), 1525.

13) M. G. Worster: Ann. Rev. Fluid Mech., 29 (1997), 91.

14) W. Yang, K. M. Chang, W. Chen, S. Mannan and J. DeBarbadillo: Metall. Mater. Trans. A, 32 (2001), 397.

15) S. D. Felicelli, J. C. Heinrich and D. R. Poirier: Metall. Trans. B, 22 (1991), 847

16) S. D. Felicelli, J. C. Heinrich and D. R. Poirier: Int. J. Num. Method. Fluids, 27 (1998), 207.

17) S. D. Felicelli, D. R. Poirier and J. C. Heinrich: J. Cry. Growth, 177 (1997), 145.

18) M. C. Schneider and C. Beckermann: Metall. Mater. Trans. A, 26 (1995), 2373.

19) J. C. Heinrich and D. R. Poirier: C. R. Mecanique, 332 (2004), 429.

20) C. Frueh, D. R. Poirier and S. D. Felicelli: Mater. Sci. Eng. A, 328 (2002), 245

21) M. G. Worster and R. C. Kerr: J. Fluid Mech., 269 (1994), 23.

22) S. D. Felicelli, D. R. Poirier and J. C. Heinrich: Metall. Mater. Trans. B, 29 (1998), 847.

23) S.M. Copley, A. F. Giamei, S. M. Johnson and M. F. Hornbecker: Metall. Trans., 1 (1970), 2193.

24) S. D. Felicelli, J. C. Heinrich and D. R. Poirier: J. Cry. Growth, 191 (1998), 879

25) R. C. Atwood and P. D. Lee: Acta Mater., 51 (2003), 5447.

26) H. B. Dong and P. D. Lee: Acta Mater, 53 (2005), 659.

27) P. D. Lee, R. C. Atwood, R. J. Dashwood and H. Nagaumi: Mater. Sci. Eng. A, 328 (2002), 213.

28) P.D. Lee, A. Chirazi, R. C. Atwood and W. Wang: Mater. Sci. Eng. A, 365 (2004), 57

29) W. Wang, P. D. Lee and M. McLean: Acta Mater., 51 (2003), 2971

30) L. Yuan, P. D. Lee, G. Djambazov and K. Pericleous: Int. J. Cast. Metal. Res., 22 (2009), 204.

31) L. Yuan, P. D. Lee, G. Djambazov and K. Pericleous: Proc. MCWASP XII, ed. by S. L. Cockcroft and D. M. Maijer, TMS, Warrendale, PA, (2009), 451.

32) L. Yuan and P. D. Lee: Modell. Simul. Mater. Sci. Eng., 18 (2010), 055008.

33) M. Rappaz and C. A. Gandin: Acta Mater., 41 (1993), 345.

34) A. J. Chorin: Math. Comput., 22 (1968), 745. 